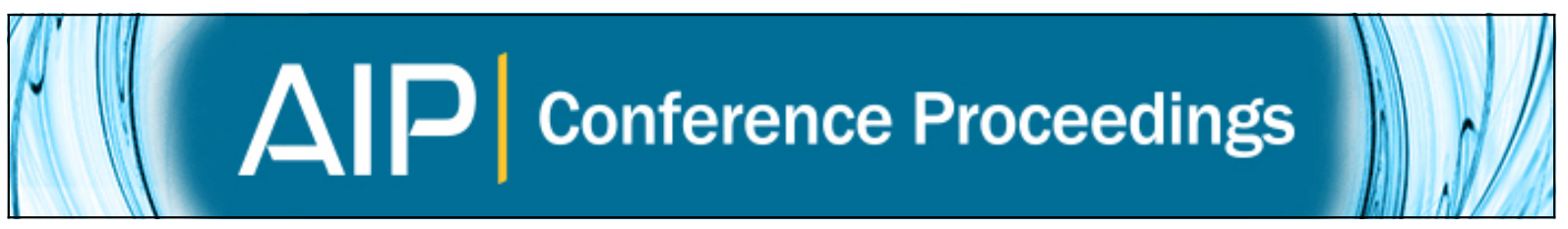

\title{
Graphene Plasmonics
}

F. Javier García de Abajo, Frank H. L. Koppens, Darrick E. Chang, and Sukosin Thongrattanasiri

Citation: AIP Conference Proceedings 1398, 28 (2011); doi: 10.1063/1.3644203

View online: http://dx.doi.org/10.1063/1.3644203

View Table of Contents: http://scitation.aip.org/content/aip/proceeding/aipcp/1398?ver=pdfcov

Published by the AIP Publishing

\section{Articles you may be interested in}

Enhanced photoluminescence of Si nanocrystals-doped cellulose nanofibers by plasmonic light scattering Appl. Phys. Lett. 107, 041111 (2015); 10.1063/1.4927672

Coupling of energy from quantum emitters to the plasmonic mode of $\mathrm{V}$ groove waveguides: A numerical study J. Appl. Phys. 111, 064323 (2012); 10.1063/1.3699031

Light Emission and Scattering in Plasmonic Nano-Structures

AIP Conf. Proc. 1398, 24 (2011); 10.1063/1.3644200

Plasmon scattering from a single gold nanoparticle collected through an optical fiber Appl. Phys. Lett. 86, 031902 (2005); 10.1063/1.1851011

Dipole and quadrupole plasmon resonances in large sodium clusters observed in scattered light J. Chem. Phys. 106, 8436 (1997); 10.1063/1.473902 


\title{
Graphene Plasmonics
}

\section{F. Javier García de Abajo, ${ }^{1,2}$ Frank H. L. Koppens, ${ }^{3}$ Darrick E. Chang, ${ }^{4}$ and Sukosin Thongrattanasiri ${ }^{1}$}

\author{
${ }^{1}$ Instituto de Optica - CSIC, Serrano 121, 28006 Madrid, Spain \\ ${ }^{3} \mathrm{ORC}$, University of Southampton, Southampton SO17 1BJ, UK \\ ${ }^{3}$ ICFO, 08860 Castelldefels (Barcelona), Spain \\ ${ }^{4}$ Caltech, Pasadena, California 9112 \\ J.G.deAbajo@csic.es
}

\begin{abstract}
Plasmons in doped graphene provide an ideal platform for strong light-matter interaction, perfect light absorption in an atomically thin layer, and ultra-large field enhancement, well beyond conventional plasmonics, and tunable through electrostatic doping.
\end{abstract}

Keywords: Plasmon, graphene, metamaterials, quantum optics.

PACS: 78.67.Wj,78.20.Ci,42.25.Bs

Graphene plasmons provide a suitable alternative to noble-metal plasmons because they exhibit much larger confinement and relatively long propagation distances, with the advantage of being highly tunable via electrostatic gating [1]. We will discuss how these properties translate into appealing optical behavior of this atomically thin material, with potential applications to infrarred detection, single-photon quantum devices, and ultrasensitive detectors. In particular, we will show that graphene layers produce extraordinarily large Pucell factors and light scattering (see Fig. 1), strong light-matter interaction, and total light absorption. These results provide the basis for the emerging and potentially far-reaching field of graphene plasmonics.
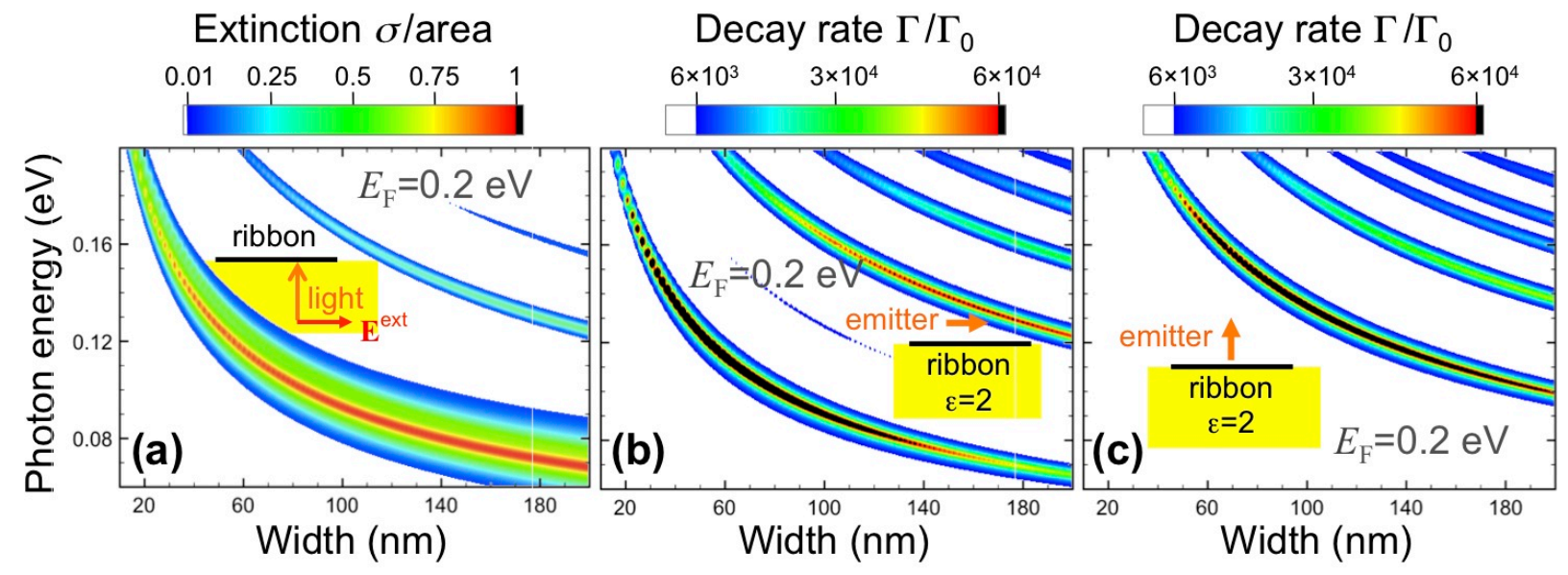

FIGURE 1. (a) Extinction cross section of doped graphene ribbons deposited on an $\varepsilon=2$ material as a function of ribbon width and photon energy for a Fermi energy $\mathrm{E}_{\mathrm{F}}=0.2 \mathrm{eV}$. The light is incident as shown in the inset. The cross section is normalized to the carbon sheet area. (b,c) Decay rate normalized to free space under the same conditions as in (a) for a line emitter situated 10 $\mathrm{nm}$ above the center of the ribbon and polarized either parallel (b) or perpendicular (c) to it. 


\section{ACKNOWLEDGMENTS}

This work has been supported in part by the Spanish MICINN (MAT2010-14885 and Consolider NanoLight.es), Fundació Cellex Barcelona, and the Euro- pean Commission (FP7-ICT-2009-4-248909-LIMA and FP7-ICT2009-4-248855-N4E). D.E.C. acknowledges support from the NSF (Grant No. PHY-0803371) and the Gordon and Betty Moore Foundation through Caltech's Center for the Physics of Information.

\section{REFERENCES}

1. F. H. L. Koppens, D. E. Chang, and F. J. García de Abajo, Nano Letters 11, 3370 (2011). 